\title{
A Case of Giant Mediastinal Liposarcoma of Thymic Origin: A Rare Clinical Entity
}

\author{
Debmalya Saha, Pawan Singh, Rakesh Sharma, Sayyed Ehtesham Hussain Naqvi, \\ Saket Aggarwal, Muhammad Abid Geelani
}

Department of Cardiothoracic \& Vascular Surgery, G.B. Pant Institute of Postgraduate Medical Education \& Research (GIPMER), New Delhi, India

Email: debmalya.cmc@gmail.com

How to cite this paper: Saha, D., Singh, P., Sharma, R., Naqvi, S.E.H., Aggarwal, S. and Geelani, M.A. (2020) A Case of Giant Mediastinal Liposarcoma of Thymic Origin: A Rare Clinical Entity. World Journal of Cardiovascular Surgery, 10, 75-82.

https://doi.org/10.4236/wjcs.2020.106010

Received: May 18, 2020

Accepted: June 16, 2020

Published: June 19, 2020

Copyright $\odot 2020$ by author(s) and Scientific Research Publishing Inc. This work is licensed under the Creative Commons Attribution International License (CC BY 4.0).

http://creativecommons.org/licenses/by/4.0/

\begin{abstract}
Thymoliposarcoma is an exceedingly rare tumor of thymus with a very few cases reported till date. This case study presents a 45 -year male with rare type of thymoma. On the contrast-enhanced CT images, there was a large mass lesion of predominantly fat attenuation in the pre-vascular compartment of the mediastinum insinuating on both sides of the visceral compartment of the mediastinum, and extending upto the bilateral cardio phrenic and anterior costophrenic angles, anterior to the right ventricle with loss of fat plane with the pericardium, with few sub-centimetric lymph nodes in the right paratracheal and AP window and a calcified right hilar lymph node, suggestive of well-differentiated liposarcoma/thymoliposarcoma. Initial CT guided tru-cut tissue biopsy was inconclusive, and the repeat biopsy revealed as fibro-collagenous tissue with area of necrosis, focal myxoid changes in the background with presence of cells which are spindle to oval in shape with mild nuclear pleomorphism and negative for S100, Cytokeratin, CD34, desmin. The entire tumor was resected en masse after meticulous dissection without the support of cardiopulmonary bypass (CPB) with an intact pericardium. Final histopathology report of the surgical biopsy specimens is consistent with dedifferentiated thymoliposarcoma with focal ganglionic cell differentiation. Postoperative follow-up CECT of thorax revealed no evidence of residual mass in the pre-vascular compartment. The patient is disease-free and asymptomatic after 6-month and he is under routine follow-up under Radiotherapy department since he received $30 \mathrm{~Gy}$ of postoperative radiotherapy (PORT).
\end{abstract}

\section{Keywords}

Thymoma, Liposarcoma, Thymoliposarcoma, Dedifferentiated 


\section{Introduction}

Thymoliposarcoma is very uncommon mesenchymal tumor usually affecting fourth to eighth decades of life. Here we will present a large resectable mediastinal liposarcoma of thymic origin with features of pressure symptoms but without any clinical evidence of thoracic compartment syndrome in a 45 -year male after a proper informed and written consent obtained from the patient.

\section{Case Report}

A 45-year nonsmoker diabetic male was referred to our OPD with complaints of chronic cough and shortness of breath for about 2 months. The general physical examination was within normal limit. No asymmetry of the chest wall was noted. Percussion notes were impaired over the basal regions of bilateral lung field with rhonchi. Breath sounds were reduced in intensity at basal regions of both sides. Other systems were normal on examination.

His routine preoperative blood investigations were normal. His Serum lactate dehydrogenase (LDH), beta-HCG and alpha fetoprotein (AFP) levels were within normal limit. His Chest X-ray (Figure 1) revealed bilateral middle and lower lobe diffuse opacification with midline trachea and no visible compression or deviation.

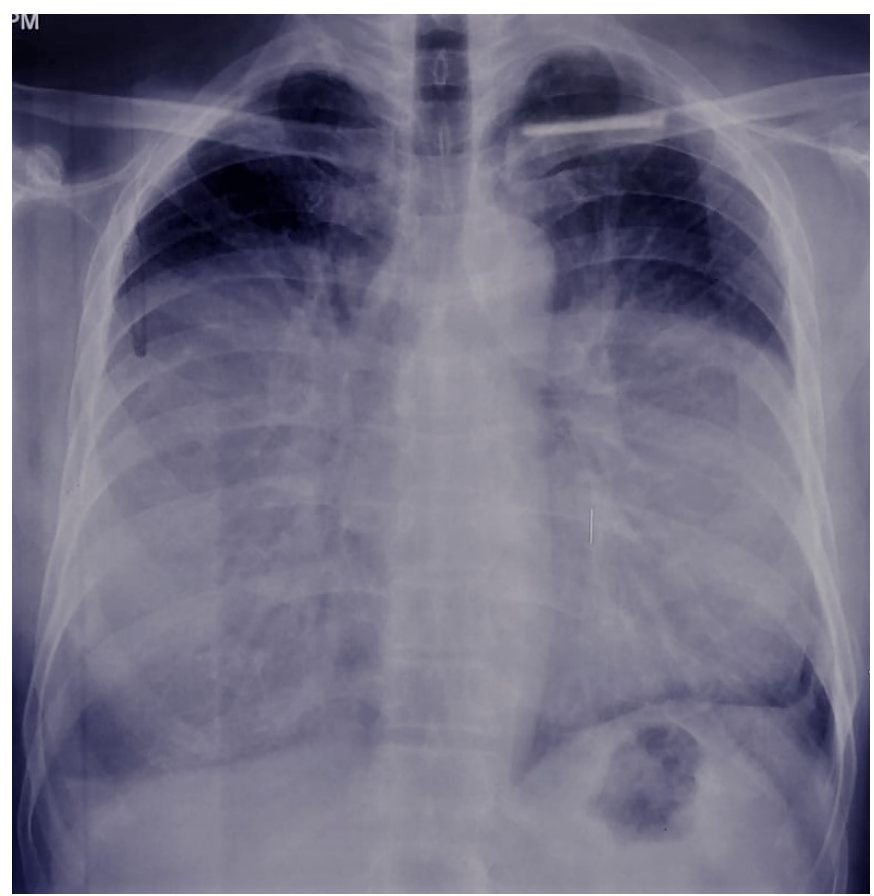

Figure 1. Preoperative Chest X-ray PA view showing bilateral middle \& lower lobe opacification.

On the contrast-enhanced CT images (Figure 2), there was a large mass lesion of predominantly fat attenuation in the pre-vascular compartment of the mediastinum insinuating on both sides of the visceral compartment of the medias- 
tinum, and extending upto the bilateral cardio phrenic and anterior costophrenic angles, anterior to the right ventricle with loss of fat plane with the pericardium, with few sub-centimetric lymph nodes in the right paratracheal and AP window and a calcified right hilar lymph node, suggestive of well-differentiated liposarcoma/Thymoliposarcoma. MRI of thorax (Figure 3) revealed large well-defined soft tissue mass in the pre-vascular and visceral compartment of mediastinum extending into bilateral hemithorax showing predominantly heterogenous fat signal with lobulated heterogeneously enhancing soft tissue component; superiorly the mass was extending till the level of clavicles, inferiorly upto diaphragmatic surface extending into right cardio phrenic and costophrenic angles, laterally upto chest walls of both sides abutting the inner surface of ribs with maintained extra pleural fat and no obvious infiltration into the overlying soft tissue; anteriorly, abutting the posterior surface of the sternum; posteriorly, it was displacing and molding around the anterior surface of cardiac chambers and major mediastinal vessels with focal loss of fat planes between solid component and the pericardium overlying the left ventricle. 18F-FDG PET-CT study revealed multiple soft tissue attenuating linear strands within the mass of the pre-vascular compartment of the mediastinum with well-defined metabolically active lobulated soft tissue density component, no other definite scan evidence of metabolically active disease was seen in the rest of the body.

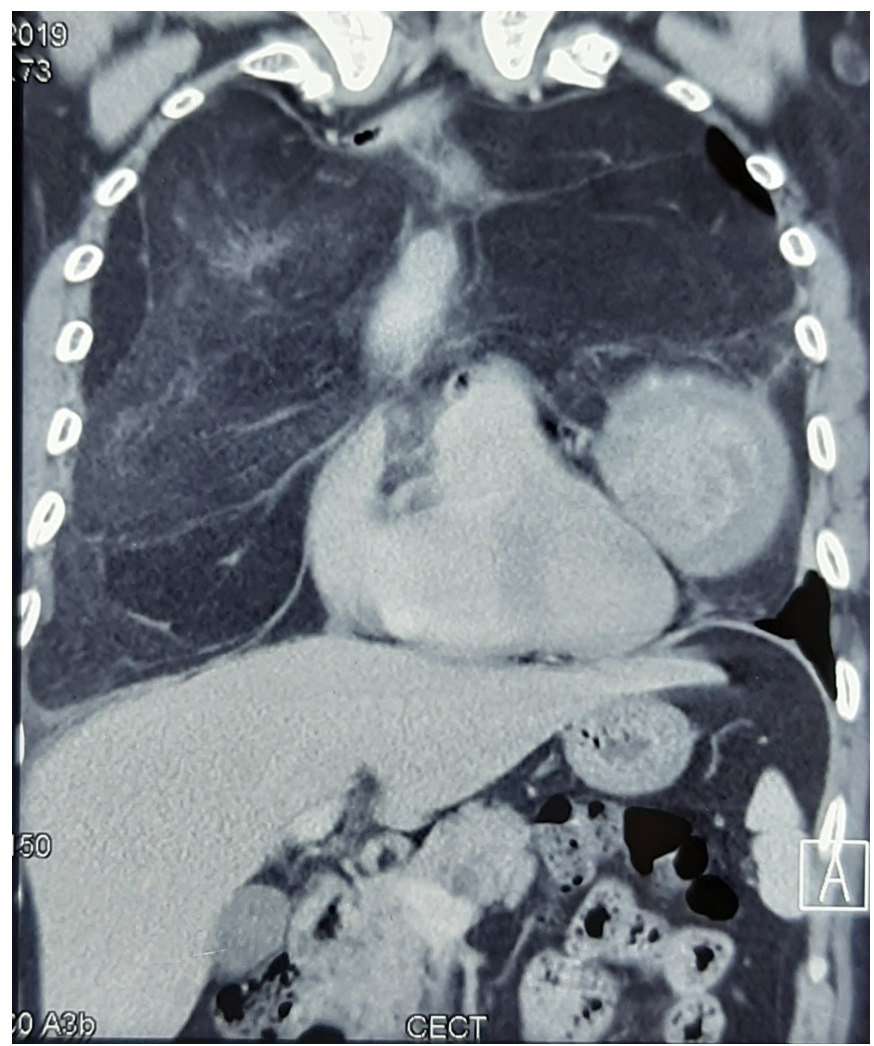

Figure 2. Preoperative CECT thorax showing a large mass lesion of predominantly fat attenuation in the mediastinum, and extending upto the bilateral cardiophrenic and costophrenic angles. 


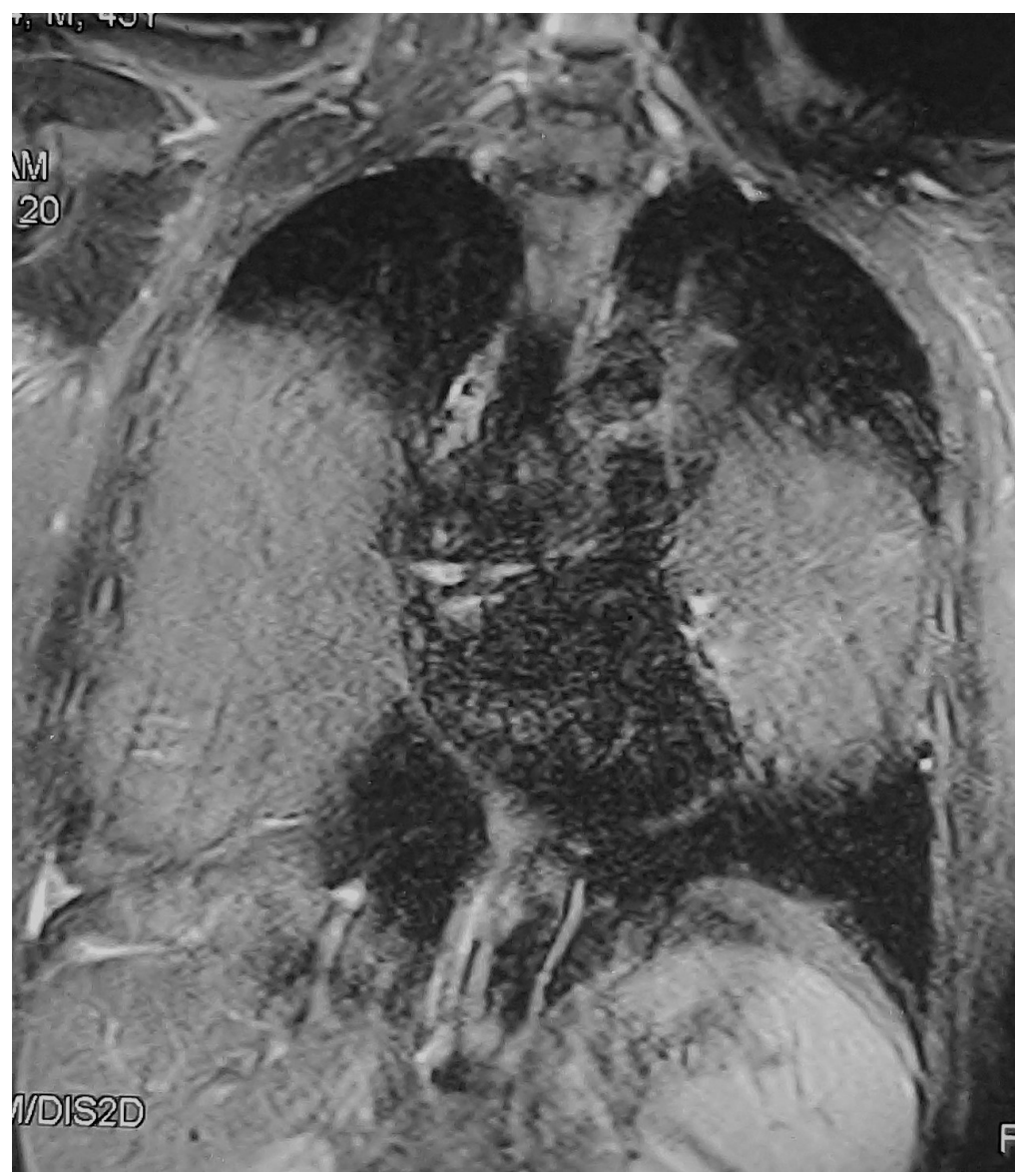

Figure 3. MRI of thorax-large well-defined soft tissue mass in the mediastinum extending into bilateral hemithorax showing predominantly heterogenous fat signal with heterogeneously enhancing soft tissue component.

Initial CT guided tru-cut tissue biopsy was inconclusive, and the repeat biopsy revealed as fibro-collagenous tissue with area of necrosis, focal myxoid changes in the background with presence of cells which are spindle to oval in shape with mild nuclear pleomorphism and negative for S100, Cytokeratin, CD34, desmin.

The patient was planned for surgical resection with curative intent. Our approach was via median sternotomy. On exploration (Figure 4), an exceptionally large bilobed thymic mass was found in anterior mediastinum, with the base adhered to the pericardium with maintained fat planes and the apex to the left brachiocephalic vein. The tumor was supplied by branches from the Left internal mammary artery (LIMA) and drained by tributaries of left brachiocephalic vein. Both lobes of the tumor were extending to the bilateral pleural spaces with compression of the underlying lungs. The entire tumor was resected en masse after meticulous dissection without the support of cardiopulmonary bypass (CPB) with an intact pericardium (Figure 5). The both lungs were inflated by ventilation manually by the anesthetist. The postoperative period was uneventful. 


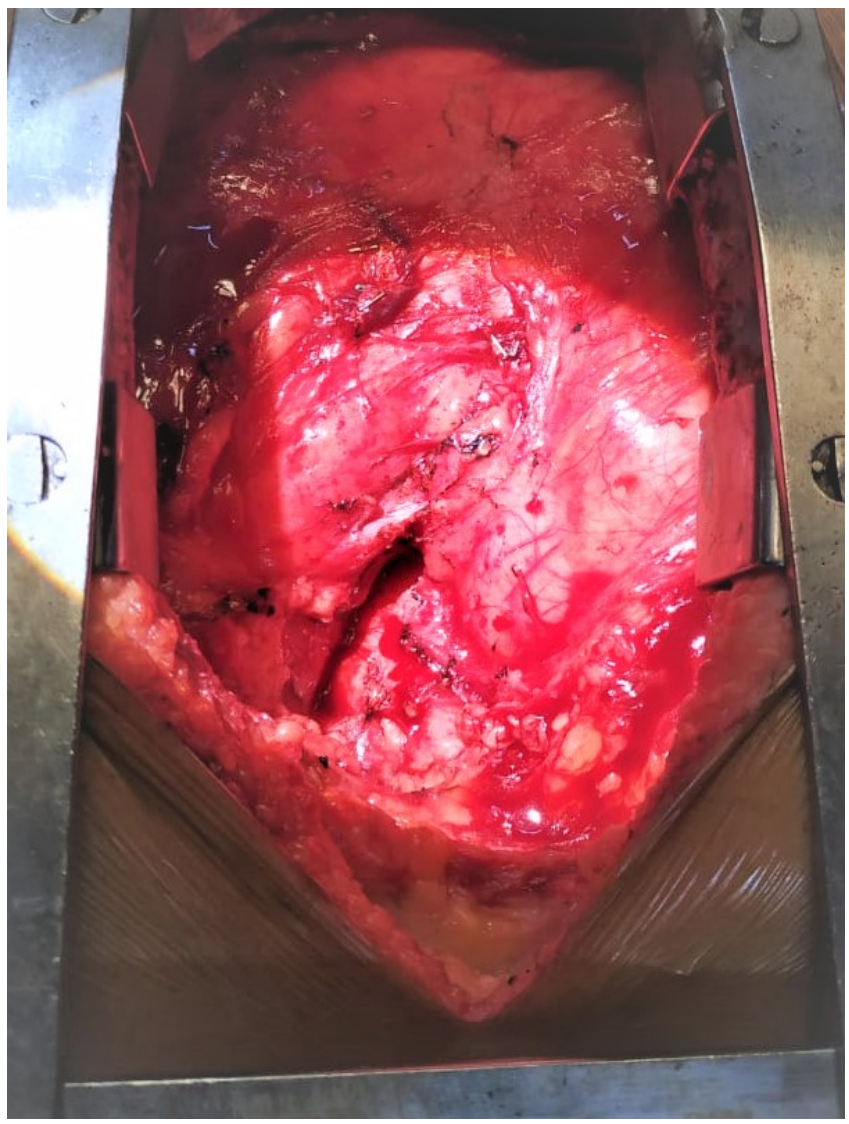

Figure 4. Large bilobed thymic mass in anterior mediastinum, with the base adhered to the pericardium.

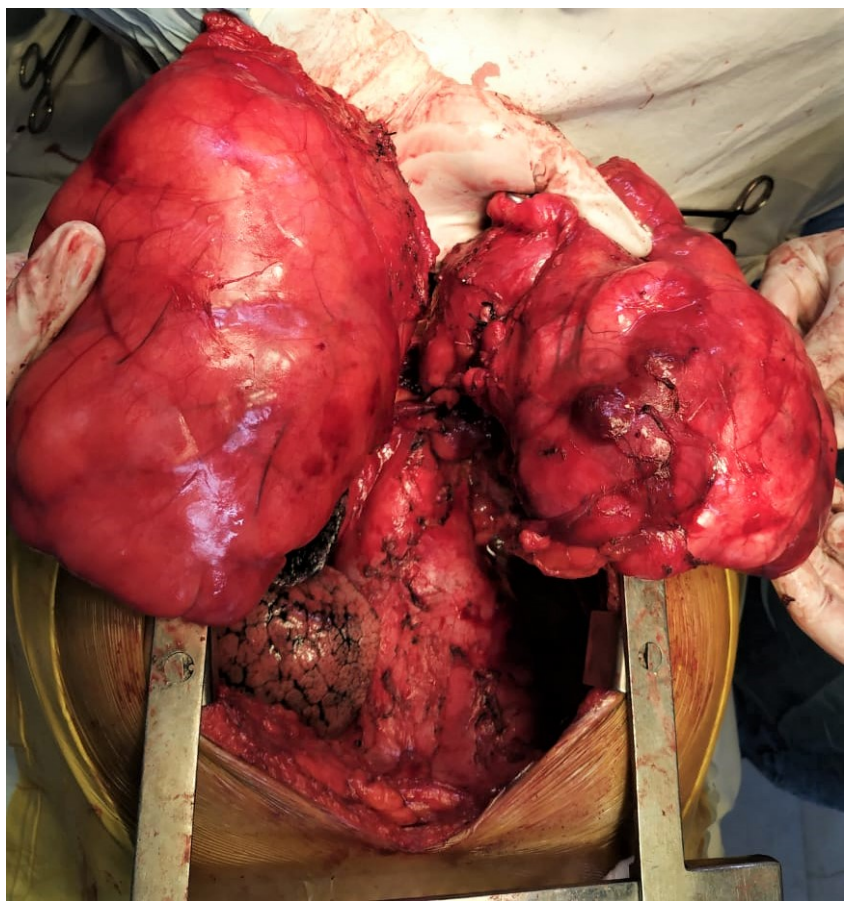

Figure 5. Entire tumor resected en masse (connecting stalk divided) with an intact pericardium. 
On gross examination (Figure 6), there was two globular encapsulated mass, right lobe measuring $18 \times 12 \times 9 \mathrm{~cm}$ and left lobe of $20 \times 12 \times 7 \mathrm{~cm}$. On serial sectioning of right lobe, multiloculated fatty areas was seen along with a single grey white nodule of $1.5 \times 1.2 \times 1.0 \mathrm{~cm}$, at some foci yellow area shows fibrous band like areas along with capsule; on cut section grey white nodule shows homogeneous areas. On serial sectioning of left lobe, there was lobulated area along with grey white nodule of $9 \times 7.5 \times 7 \mathrm{~cm}$ with necrotic changes, nodule is present at the periphery of the mass. Multiple sections were taken from both the masses and they show the variegated tumor which at place is composed of mature adipose tissue interspersed with lipoblast; at other place, tumor was is composed of sheets \& fascicles, whorls of spindle cells, large areas of necrosis intermixed with thymic tissue; the spindle cells are separated by delicate fibrovascular channels; numerous variably large round cells are identified with large vesicular nuclei, prominent nucleoli and abundant eosinophilic cytoplasm containing melanin pigment (Figure 7). On immunohistochemistry, the spindle cells are negative for S100, SMA, synaptophysin and chromogranin. The features of both right and left mediastinal mass biopsy are consistent with dedifferentiated thymoliposarcoma with focal ganglionic cell differentiation.

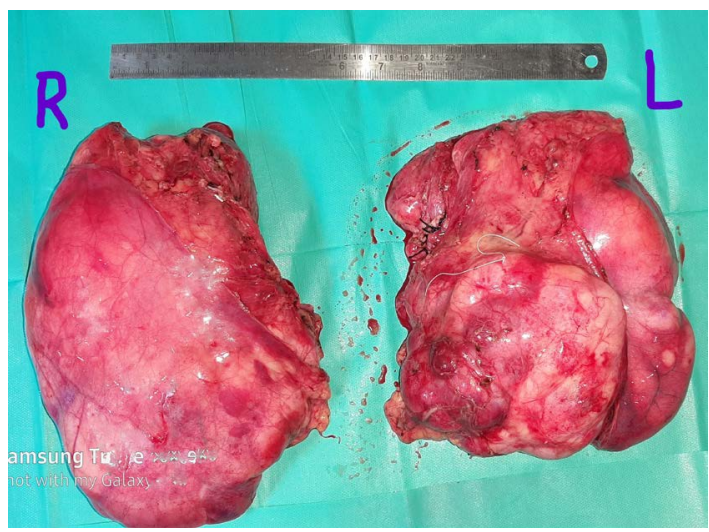

Figure 6. Gross pictures of two globular encapsulated masses (ex-vivo).

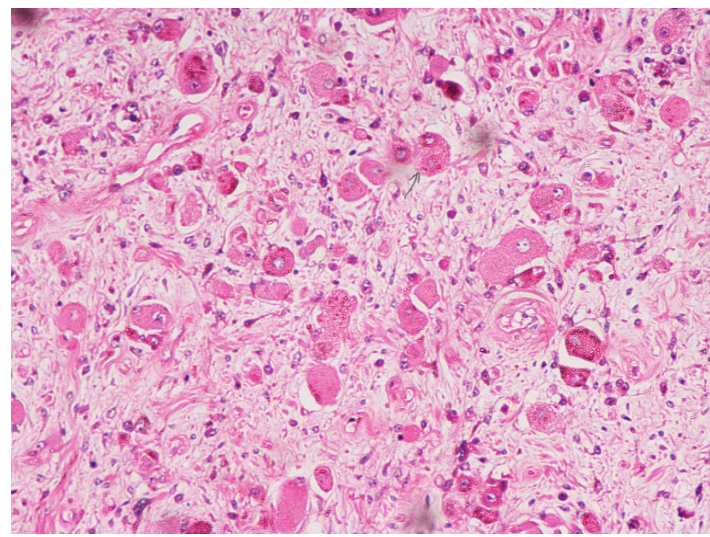

Figure 7. Dedifferentiated liposarcoma with ganglionic cell differentiation. 
Postoperative follow-up CECT of thorax (Figure 8) revealed no evidence of residual mass in the pre-vascular compartment of the mediastinum. The patient is disease-free and asymptomatic after 6-month and he is under routine follow-up under Radiotherapy department since he received 30Gy of postoperative radiotherapy (PORT).

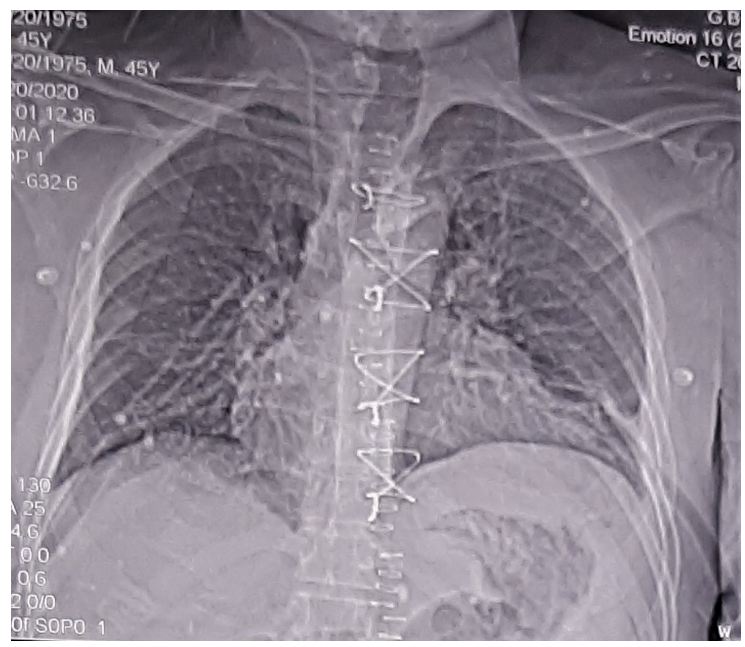

Figure 8. Postoperative follow-up CECT of thorax showing no evidence of residual mass in the mediastinum with expanded bilteral lung fields.

\section{Discussion}

Liposarcomas of thymic origin i.e. thymoliposarcoma is very rare entity of mesenchymal tumor mainly affecting fourth to eighth decades of life with a median age of 58 years with a slight female predominance $(\mathrm{M}: \mathrm{F}=1: 2)$ [1] [2] [3] [4]. The tumors are usually large in size ( 9 to $13 \mathrm{~cm}$, average $11.6 \mathrm{~cm}$ ) at the time of diagnosis, in spite of this fact that the patients may remain symptom-free or [1] [4] may come to clinic with nonspecific symptoms [2] [3]. An incidentally diagnosed mediastinal mass detected by chest skiagram done for some unrelated problems is the scenario mostly. The symptom-complex is due to the expansile character of the tumor rather than infiltrative nature, with lower propensity to involve the surrounding vital structures.

On gross examination, these tumors are mostly encapsulated, lobulated, and with presence of yellowish adipose, whitish fibrous, and sometimes myxoid components. Thymoliposarcomas are broad spectrum tumors, including sclerosing, lipomatous, myxofibrosarcomatous, and dedifferentiated subtypes histologically. The intervening thymic tissue in the sarcoma is the ominous feature to differentiate from the other mediastinal liposarcomas. A dedifferentiated thymoliposarcoma contains well-differentiated lipoblasts with areas of high-grade sarcomatous cells (non-lipogenic) with variable degree of necrosis, anaplasia, and mitosis. Morphologic differences are seen in different areas of the same tumor as well as between the primary and secondary lesion of the same patient [3]. Pleo- 
morphic variety has more aggressive outcome than well-differentiated or dedifferentiated liposarcoma. According to genetic analysis, chromosomal amplification of $12 \mathrm{q} 14$ to 15 regions, including MDM2 gene is characteristic feature of well-differentiated or dedifferentiated liposarcomas, but pleomorphic liposarcomas lack this genetic alteration [5].

The thymolipoma, thymofibroma, thymofibrolipoma, Hodgkin lymphoma, inflammatory pseudotumor as well as sclerosing mediastinitis are the differential diagnosis of thymoliposarcomas. Like thymoliposarcomas, thymolipomas usually affect younger patients in their third decade as an incidentally diagnosed large anterior mediastinal mass with histologically composed of thymic tissue and mature adipocytes but without the lipoblasts. There is no association between myasthenia gravis and thymoliposarcoma as it is reported in thymolipoma [5] [6]. The distinguishing feature between well-differentiated thymoliposarcoma and thymofibrolipoma is lack of atypical lipoblast in thymofibrolipoma. The characteristic CD30 and CD15 positive lacunar or Reed-Sternberg cells in nodular sclerosing Hodgkin's lymphoma differentiate them from thymoliposarcoma.

Thymoliposarcomas usually have a benign clinical course except the dedifferentiated variety; neither histologically proven metastasis nor disease-specific mortality is common. The mainstay of treating thymoliposarcoma is surgery. Adjuvant radiotherapy has some role both in primaries as well as recurrence.

\section{Conflicts of Interest}

The authors declare no conflicts of interest regarding the publication of this paper.

\section{References}

[1] Sekine, Y., Hamaguchi, K., Miyahara, Y., et al. (1996) Thymus-Related Liposarcoma: Report of a Case and Review of the Literature. Surgery Today, 26, 203-207. https://doi.org/10.1007/BF00311509

[2] Howling, S.J., Flint, J.D.A. and Muller, N.L. (1999) Thymoliposarcoma: CT and Pathologic Findings. Clinical Radiology, 54, 341. https://doi.org/10.1016/S0009-9260(99)90571-8

[3] Havlicek, F. and Rosai, J. (1984) A Sarcoma of Thymic Stroma with Features of Liposarcoma. American Journal of Clinical Pathology, 82, 217-224. https://doi.org/10.1093/ajcp/82.2.217

[4] Klimstra, D.S., Morgen, C.A., Perino, G., Koss, M.N. and Rosai, J. (1995) Liposarcoma of the Anterior Mediastinum and Thymus. The American Journal of Surgical Pathology, 19, 782-791. https://doi.org/10.1097/00000478-199507000-00006

[5] Fletcher, C.D.M., Unni, K.K. and Mertens, F. (2002) WHO Classification Tumors of Soft Tissue and Bone. IARC Press, Lyon, 35-45.

[6] Pan, C.H., Chiang, C.Y. and Chen, S.S. (1988) Thymolipoma in Patients with Myasthenia Gravis: Report of Two Cases and Review. Acta Neurologica Scandinavica, 78, 6-21. https://doi.org/10.1111/j.1600-0404.1988.tb03612.x 